\title{
Medizin und Lachen
}

\author{
Lachen tut gut - das weiss jeder. Aber hilft es auch heilen? Einige Studien sagen ja: \\ es stärke das Immunsystem, wirke sich positiv auf das kardiovaskuläre System aus, \\ erhöhe die Schmerzschwelle, bewirke die Abnahme des Muskeltonus und führe zu er- \\ höhter peripherer Sauerstoffsättigung des Blutes.
}

\section{Tabea Gubler}

Die Autorin studiert Humanmedizin im 4. Studienjahr an der Universität Zürich und hat das Thema im Frühjahrssemester 2010 im Rahmen des Mantelstudium-Moduls

«Medizingeschichte» bearbeitet.
Jedesmal, wenn ein Mensch lacht, fügt er seinem Leben ein paar Tage hinzu.

(Curzio Malaparte)

Lachen ist gesund. Kaum jemand wird dem nicht spontan zustimmen. Wohl jeder hat schon einmal erlebt, wie ein herzliches Lachen guttun kann. Lachen ist eine Grundausdrucksform des Menschen. Grundsätzlich verbinden wir es mit positiven Gefühlen und mit Zugehörigkeit. So lag schon immer der Gedanke nahe, dass Lachen sich positiv auf den Körper und die Psyche auswirkt. «Zwischen Lachen und Spielen werden die Seelen gesund» ist ein Sprichwort aus Arabien $[1]^{\star}$. In Angola sagt man: «Lachen reinigt die Zähne» [2]. Und auch in der Bibel heisst es: «Ein fröhliches Herz bringt gute Besserung, aber ein niedergeschlagener Geist dörrt das Gebein aus» (Sprüche 17, 22).

Die Stellung des Humors in der Medizin ist vielfältig. Reden wir über den Humor in der Beziehung zwischen Arzt und Patient oder geht es um Humor als spezifische Therapie durch ausgebildete «Humortherapeuten»? Geht es um die Spitalclowns? Sprechen wir vom Humor bei Kindern, geriatrischen Patienten oder psychisch Kranken? An dieser Stelle soll der Frage nachgegangen werden, wie der Humor den Weg in die Wissenschaft und in die heutige Medizin gefunden hat.

\section{Die Entwicklung der «Lachforschung»}

1964 wurde von William F. Fry das erste Institut der Gelotologie (also der «Lachwissenschaft») an der Stanford Universität in Kalifornien gegründet. Wegen mangelnder Unterstützung finanzierte er das Institut aus eigenen Mitteln [3]. Der Zeitpunkt ist vermutlich kein Zufall. Die Welt, auch die Wissenschaft, war im Wandel. Die Psychologie wendete sich ab vom «Behaviorismus», also von der Ansicht, dass das Verhalten von Menschen mit den Methoden der Naturwissenschaft untersucht werden kann, und hin zum «Kognitivismus», einer Richtung, bei der den innerpsychischen Vorgängen Beachtung geschenkt wurde [4].

Das Interesse für die Gelotologie hängt auch mit der vertieften Forschung über psychosomatische Aspekte zusammen. In der Medizin entwickelte sich in dieser Zeit diese neue Fachrichtung. 1942 wurde die American Psychosomatic Society gegründet [5]. Die
Schweiz war später mit dabei. In Zürich etwa wurde 1980 an der Psychiatrischen Poliklinik die Abteilung für Psychosoziale Medizin unter der Leitung von Prof. Jürg Willi eröffnet [6].

Eine Voraussetzung speziell für die Entstehung der Spitalclowns war wohl auch die Forschung zum «Hospitalismus», also den negativen Folgen von längeren Krankenhausaufenthalten, insbesondere bei Kindern. H. M. Skeels, ein US-amerikanischer Psychologe, untersuchte in den 1940er Jahren die Auswirkungen von mangelnder emotionaler Zuwendung in Heimen auf die kindliche Entwicklung [7]. Dies hatte einen starken Einfluss auf das Entstehen der Spitalclowns, speziell in der Pädiatrie.

1979 erregte der autobiographische Krankheitsbericht des US-Wissenschaftsjournalisten Norman Cousins Aufsehen: Er beschreibt in «Anatomy of an Illness» (deutsch: «Der Arzt in uns selbst», 1981), wie sich seine Spondylarthritis durch eine selbst verordnete «Lachtherapie» verbesserte. Durch lustige Filme und Bücher brachte er sich zum Lachen, seine Schmerzen liessen unmittelbar nach, er konnte nun eine Stunde am Stück schlafen, und die Blutsenkungsgeschwindigkeit nahm jeweils ab. Auch durch die zahlreichen Reaktionen auf dieses Buch erlebten die Gelotologie sowie Spitalclowns einen Aufschwung.

\section{Spital- und andere Clowns}

Der US-amerikanische Arzt Patch Adams zählt zu den Pionieren des Lachens in der Medizin. Bekannt ist die Verfilmung seiner Geschichte (1998) unter dem gleichnamigen Titel «Patch Adams» mit Robin Williams. Bereits 1972 hatte er das «Gesundheit!-Institute» gegründet. Die Zielsetzung ist eine kostenlose ganzheitliche Behandlung, bei der der Humor eine grosse Rolle spielt und Patienten als «Freunde» behandelt werden [8].

Die ersten Spitalclowns gab es 1986 in New York [9]. Bei dem Fest eines Kinderkrankenhauses traten Clowns des «Big Apple Circus» mit weissem Kittel und roter Nase auf. So entstand die Idee der «Clown Care Unit», Clowns, die Kinder im Spital besuchen [10].

Spitalclowns sind heute in der Pädiatrie weitverbreitet, 47 Spitäler und Institutionen der Schweiz werden von Spitalclowns der Stiftung Theodora besucht [11]. Auch in der Geriatrie wurden bereits Spitalclowns bei Patienten mit Demenz eingesetzt [12]. 
Seit knapp über 3 Jahren besuchen Clowns des schweizerischen Vereins «Huusglön» Menschen mit körperlichen und geistigen Behinderungen oder mit schwerwiegenden Erkrankungen zu Hause*. Inspiriert zu der Idee wurden die Huusglön durch einen gemeinsamen Einsatz mit Patch Adams in Russland. Nach einem Projektjahr wurde der Verein 2008 gegründet. Das Ziel ist, «diesen Menschen etwas Fröhlichkeit ins Haus zu bringen», wie es auf der Homepage heisst [13]. Die Huusglön erweitern damit das Konzept der Spitalclowns auf Hausbesuche und beschränken sich nicht nur auf Kinder.

\section{Kann man das Lachen als Therapie verschreiben?}

\section{Lachyoga}

Eine Organisation der speziellen Art, nämlich den ersten Lachclub mit Lachyogaübungen, gründete der indische Arzt Dr. Madan Kataria im Jahre 1995. Inspiriert zu dieser Idee hatte ihn unter anderem Norman Cousins Buch. Heute gibt es über 6000 Lachclubs weltweit und einen Weltlachtag (1. Sonntag im Mai) [14]. Die Idee des Lachyogas ist es, dass auch grundloses Lachen hilft. Die Wirkungen des Lachens auf den Körper seien unabhängig vom Grund des Lachens.

\section{Humor: eine evidenzbasierte Therapie?}

Seit den 1990er Jahren ist die Evidence-Based Medicine das medizinische Legitimationskonzept. Heute gibt es daher auch zahlreiche Studien über die Wirkungen des Lachens auf den Körper. Lachen soll unter anderem das Immunsystem stärken (durch Aktivierung der natürlichen Killerzellen $[15,16])$ und sich positiv auf das kardiovaskuläre System auswirken (durch vermehrte Vasodilatation wegen erhöhter Ausschüttung von beta-Endorphinen und NO [17], bzw. durch eine auf das Lachen folgende Periode von erniedrigter Herzfrequenz und erniedrigtem Blutdruck [18]). Weitere postulierte Effekte sind die Erhöhung der Schmerzschwelle [19], die Abnahme des Muskeltonus [20] und eine erhöhte periphere Sauerstoffsättigung des Blutes [21]. Allerdings gibt es Reviews, die die Ergebnisse der Studien anzweifeln. [22, 23] Nicht zuletzt gilt der Satz von Mark Twain: «Studying humour is like dissecting a frog - you may know a lot but you end up with a dead frog.»

Da Lachen im Prinzip keine Nebenwirkungen hat, stellen manche Autoren die Frage, ob man beim Lachen die gleichen Kriterien für die Evidenz anwenden kann wie beispielsweise für ein neues Medikament [24]. Wegen mangelnder Evidenz vom Lachen abzuraten, macht wenig Sinn, und dass das Lachen zumindest psychisch guttut, muss man wohl nicht beweisen.

Die Frage ist nur: Kann man das Lachen als Therapie verschreiben im Sinne von: «Lachen Sie ab heute häufiger!»? Lachen lasse sich zwar nicht erzwingen, aber sei erlernbar, so der US-Psychologe Paul McGhee. Am Psychologischen Institut der Universität Zürich läuft momentan eine Studie, bei der das adaptierte Humortraining von McGhee an Testpersonen durchgeführt wird, und die Wirkungen wissenschaftlich ausgewertet werden [25]. Die Arbeit gehört zum dortigen Forschungsschwerpunkt der positiven Psychologie. In diesem Sommer fand in Zürich bereits die zehnte «International Summer School and Symposium on Humour and Laughter» statt [26]. Wenn Humor erlernbar ist und präventiv oder gar therapeutisch wirkt, wäre eine ärztlich verordnete Humortherapie tatsächlich denkbar. Ein Training kann auch für Ärzte sinnvoll sein, da man als Arzt mit unsensiblem Humor auch einiges falsch machen kann. Der Einsatz von Humor in der Therapie will gelernt sein.

\section{Blick in die Zukunft}

Die Frage bleibt offen, wie sich der Stellenwert des Humors in der Medizin entwickeln wird. Wird der Aspekt des Humors in der Arzt-Patienten-Beziehung zum Lehrstoff des Medizinstudiums? Werden mehr spezifisch ausgebildete Clowns eingesetzt werden - im Spital, im Heim und zu Hause? Werden Ärzte den Patienten Lachyoga oder Humortrainings verschreiben? Oder wird es gar im Rahmen der Weiterbildung «Humortrainings» für Ärzte geben? Es bleibt spannend. In einer Zeit, in der der Anteil chronisch kranker Patienten steigt und die Prävention und das Anleiten zu einem gesunden Lebensstil als immer wichtiger erachtet werden, ist ein ressourcenorientierter Ansatz zentral. Der Humor kann dabei eine relevante Rolle spielen.

Komik und Lachen begegnen uns sozusagen als Schwimmring, der die Menschen trotz tragischer oder widriger Umstände - sei dies im Schauspiel oder im realen Leben - vor dem Untergehen bewahrt. (Iren Bischofberger [27])
Die «Huusglön» besuchen Menschen mit Behinderungen und chronischen Erkrankungen in ihrem Zuhause und bringen etwas Fröhlichkeit. 


\section{Literatur}

1 www.journalistenakademie.de/ dossierbeitrag.php?b=1022, 2.7.2010

2 www.bk-luebeck.eu/ sprichwoerter-afrikanische-3.html, 2.7.2010

3 http://de.wikipedia.org/wiki/William_F._Fry. 2.7.2010

4 http://de.wikipedia.org/wiki/Kognitive_Wende, 2.7.2010

5 www.psychosomatic.org/about/index.htm, 2.7.2010

6 www.psychiatrie.usz.ch/UeberUns/Geschichte/ Seiten/Geschichte2.aspx, 2.7.2010

7 http://de.wikipedia.org/wiki/Harold_M._Skeels, 2.7.2010

8 www.patchadams.org/mission. Gesundheit's Mission. 3.7.2010

9 www.bigapplecircus.org/community/ clown-care.aspx. Clown Care. 3.7.2010

10 Görke, S. Klinikclowns: www.humorcare.com/ informationen/texte/klinikclowns.html, 3.7.2010

11 Poulie, A. Geschichte. www.theodora.ch/ index.php?pageid=184. 3.7.2010

12 Rösner, M. Der Gericlown, eine Vorstellung. Z Gerontol Geriatr. 2010 Feb;43(1):53-7. Epub 2009 Dec 25

13 www.huusgloen.ch/index_de.html. Idee und Zielsetzung. 2.7.2010

14 The History of Laughter Yoga. www.laughteryoga.org/ index.php?option=com_content\&view=article\&id= 460: history-of-laughter-yoga\&catid=85:aboutlaughter-yoga\&Itemid=265 3 . $7 \cdot 2010$

15 Bennett MP, Zeller JM, Rosenberg L, McCann J. The effect of mirthful laughter on stress and natural killer cell activity. Altern Ther Health Med. 2003;9(2):38-45.

16 Berk LS, Felten DL, Tan SA, Bittman BB, Westengard J. Modulation of neuroimmune parameters during the eustress of humorassociated mirthful laughter. Altern Ther Health Med. 2001 Mar;7(2):62-72, 74-6.
17 Miller M, Fry WF. The effect of mirthful laughter on the human cardiovascular system. Med Hypotheses. 2009 Nov;73(5):636-9. Epub 2009 May 27.

18 Bennett MP, Lengacher C. Humor and Laughter May Influence Health: III. Laughter and Health Outcomes. Evid Based Complement Alternat Med. 2008 Mar; 5(1):37-40.

19 Zweyer K, Velker B, Ruch W. Do cheerfulness, exhilaration and humour production moderate pain tolerance? A FACS study. In R.A. Martin (Ed.), Sense of Humor and Health [special issue]. Humor: International Journal of Humor Research, 2004;17, 67-84.

20 Paskind J. Effects of laughter on muscle tone. Arch Neurol Psychiatry.1932;28:623-8.

21 Fry WF Jr, Stoft PE. Mirth and oxygen saturation levels of peripheral blood. Psychother Psychosom. 1971;19(1):76-84.

22 Martin RA. Humor, laughter, and physical health: methodological issues and research findings. Psychol Bull. 2001;127(4):504-19.

23 McCreaddie M, Wiggins S. The purpose and function of humour in health, health care and nursing: a narrative review. J Adv Nurs. 2008 Mar;61(6):584-95.

24 Strean WB. Laughter prescription. Can Fam Physician. 2009 Oct;55(10):965-7.

25 Rusch S, Stolz H. Humortraining. www.psychologie. uzh.ch/perspsy/trainings/humortraining.php. 3.7.2010

26 www.humoursummerschool.org/. 2.7.2010

27 Bischofberger I. Stell dir vor, du bist krank und keiner hat Humor! www.humorcare.com/ informationen/texte/dubistkrank.html. 3.7.2010 